\title{
A spectral mapping theorem for Banach modules
}

\author{
by \\ H. SEFEROĞLu (Turkey)
}

\begin{abstract}
Let $G$ be a locally compact abelian group, $M(G)$ the convolution measure algebra, and $X$ a Banach $M(G)$-module under the module multiplication $\mu \circ x, \mu \in M(G)$, $x \in X$. We show that if $X$ is an essential $L^{1}(G)$-module, then $\sigma\left(T_{\mu}\right)=\widehat{\widehat{\mu}(\operatorname{sp}(X))}$ for each measure $\mu$ in $\operatorname{reg}(M(G))$, where $T_{\mu}$ denotes the operator in $B(X)$ defined by $T_{\mu} x=\mu \circ x$, $\sigma(\cdot)$ the usual spectrum in $B(X), \operatorname{sp}(X)$ the hull in $L^{1}(G)$ of the ideal $I_{X}=\{f \in$ $\left.L^{1}(G) \mid T_{f}=0\right\}, \widehat{\mu}$ the Fourier-Stieltjes transform of $\mu$, and $\operatorname{reg}(M(G))$ the largest closed regular subalgebra of $M(G) ; \operatorname{reg}(M(G))$ contains all the absolutely continuous measures and discrete measures.
\end{abstract}

1. Introduction. Let $G$ be a locally compact abelian group, $\widehat{G}$ its dual group, $L^{1}(G)$ the group algebra of $G$, and $M(G)$ the Banach algebra of all bounded regular complex Borel measures on $G$. It is well known that $M(G)$ is a commutative Banach algebra with the identity $\delta_{0}$, where $\delta_{0}$ is the Dirac measure concentrated in zero. It follows from Albrecht's theorem [1] that there exists a largest closed regular subalgebra of $M(G)$. As in [11] we denote this algebra by $\operatorname{reg}(M(G))$. Since the group algebra $L^{1}(G)$ and the discrete measure algebra $M_{\mathrm{d}}(G)$ are regular Banach subalgebras of $M(G)$, we have $L^{1}(G)+M_{\mathrm{d}}(G) \subset \operatorname{reg}(M(G))$. But in general, $L^{1}(G)+M_{\mathrm{d}}(G) \neq \operatorname{reg}(M(G))$ (see [11]). Furthermore, $\widehat{G}$ can be considered as a subset of the structure space of $\operatorname{reg}(M(G))$, and the restriction of the Gelfand transform of $\mu \in \operatorname{reg}(M(G))$ to $\widehat{G}$ coincides with the FourierStieltjes transform $\widehat{\mu}$ of $\mu$. Note also that $\operatorname{reg}(M(G))$ is a semisimple algebra with the identity $\delta_{0}$.

Let $X$ be a Banach space, $B(X)$ the algebra of all bounded linear operators on $X$, and $1_{X}$ the unit element of $B(X)$. For any $T \in B(X)$ we denote by $\sigma(T)$ the spectrum of $T$. For any (continuous) representation $U$ of $G$ by isometries on $X$ and for any $\mu \in M(G)$ the generalized convolution operator $\pi(\mu) \in B(X)$ is defined by

2000 Mathematics Subject Classification: Primary 46H15; Secondary 47A10.

Key words and phrases: Banach modules, Banach algebras, spectrum, Fourier-Stieltjes transform. 


$$
\pi(\mu)=\int_{G} U(g) d \mu(g) .
$$

The Arveson spectrum $\operatorname{sp}(U)$ of $U$ (see [2]) is defined as the hull in $L^{1}(G)$ of the closed ideal $I_{U}=\left\{f \in L^{1}(G) \mid \pi(f)=0\right\}$. In this setting, A. Connes [4] proved that for every Dirac measure $\mu$ the spectral mapping theorem

$$
\sigma(\pi(\mu))=\overline{\widehat{\mu}(\operatorname{sp}(U))}
$$

holds. C.D'Antoni, R. Longo and L. Zsidó [5] proved the spectral mapping theorem for every $\mu \in L^{1}(G)+M_{\mathrm{d}}(G)$. Also, S.-E. Takahasi and J. Inoue [11] proved the spectral mapping theorem for any $\mu \in \operatorname{reg}(M(G))$ in the case that $G$ is compact.

Since $\operatorname{reg}(M(G)) \supsetneq L^{1}(G)+M_{\mathrm{d}}(G)$, the Takahasi-Inoue theorem contains the D'Antoni-Longo-Zsidó spectral mapping theorem for the compact case. However, the spectral mapping theorem is not true for every $\mu \in M(G)$ ([5, Remark 1]).

Now, let $X$ be a Banach $M(G)$-module under the module multiplication $\mu \circ x, \mu \in M(G), x \in X$. Throughout this note we will assume that $X$ is an essential $L^{1}(G)$-module, that is, the linear manifold spanned by $\{f \circ x \mid$ $\left.f \in L^{1}(G), x \in X\right\}$ is dense in $X$. This is equivalent to the following ([8, Proposition 3.4]): If $\left(e_{\alpha}\right)$ is a bounded approximate identity for $L^{1}(G)$, then $e_{\alpha} \circ x \rightarrow x$ for every $x \in X$.

For any $\mu \in M(G)$, define $T_{\mu} \in B(X)$ by $T_{\mu} x=\mu \circ x(x \in X)$. We define the spectrum $\operatorname{sp}(X)$ of $X$ as the hull in $L^{1}(G)$ of the ideal $I_{X}=\{f \in$ $\left.L^{1}(G) \mid T_{f}=0\right\}$. More precisely,

$$
\operatorname{sp}(X)=\left\{\chi \in \widehat{G} \mid T_{f}=0 \Rightarrow \widehat{f}(\chi)=0, f \in L^{1}(G)\right\},
$$

where $\widehat{f}$ denotes the Fourier transform of $f \in L^{1}(G)$. It is easily seen that $\operatorname{sp}(X)$ is a nonempty closed subset of $\widehat{G}$ whenever $X \neq\{0\}$.

2. Main result. With the above notations, our main theorem can be stated as follows.

THEOREM 2.1. If $X$ is a Banach $M(G)$-module and an essential $L^{1}(G)$ module, then

$$
\sigma\left(T_{\mu}\right)=\overline{\widehat{\mu}(\operatorname{sp}(X))} \quad \text { for all } \mu \in \operatorname{reg}(M(G)) .
$$

Note that the generalized convolution operators $\pi(\mu), \mu \in M(G)$, define the $M(G)$-module multiplication on $X$ given by $\mu \circ x=\pi(\mu) x$. It is also evident that if $\left(e_{\alpha}\right)$ is a bounded approximate identity for $L^{1}(G)$, then $\pi\left(e_{\alpha}\right) x \rightarrow x(x \in X)$. Hence $X$ is an essential $L^{1}(G)$-module under the module multiplication defined above. Thus, the above theorem contains the preceding spectral mapping theorems ([4], [5], [11]).

For the proof of the theorem we need some preliminary results. 
Let $A$ be a (complex) commutative, regular and semisimple Banach algebra, $\Delta(A)$ the structure space of $A$, and $\widehat{a}$ the Gelfand transform of $a \in A$. It is well known that for a closed subset $S$ of $\Delta(A), I(S)=\{a \in A \mid \widehat{a}(\varphi)=0$, $\varphi \in S\}$ is the largest and $J(S)=\operatorname{cl}\{a \in A \mid \operatorname{supp} \widehat{a}$ is compact and supp $\widehat{a} \cap S=\emptyset\}$ the smallest closed ideal of $A$ whose hull is $S$. For brevity, the structure space of $\operatorname{reg}(M(G))$ will be denoted by $\Delta_{\text {reg. }}$. The hull in $\operatorname{reg}(M(G))$ of the ideal $K=\left\{\mu \in \operatorname{reg}(M(G)) \mid T_{\mu}=0\right\}$ will be denoted by $h(K)$. Also the symbol $\mu^{v}$ will be used to denote the Gelfand transform of any $\mu \in \operatorname{reg}(M(G))$.

Lemma 2.2. Suppose the hypotheses of Theorem 2.1 are satisfied. Then, under the above notations,

$$
\sigma\left(T_{\mu}\right)=\mu^{v}(h(K)) \quad \text { for all } \mu \in \operatorname{reg}(M(G)) .
$$

Proof. Denote by $A$ the (operator-norm) closure of $\left\{T_{\mu} \mid \mu \in \operatorname{reg}(M(G))\right\}$. Since $X$ is an essential $L^{1}(G)$-module, from the equality $T_{\delta_{0}} T_{f}=T_{f}$ we get $T_{\delta_{0}}=1_{X}$. Thus, $A$ is a commutative unital subalgebra of $B(X)$. Consider the mapping $\theta: \Delta(A) \rightarrow h(K)$ defined by $\theta(\varphi)(\mu)=\widehat{T}_{\mu}(\varphi)$. First we show that $\theta$ is onto (since $\theta$ is one-to-one, this means that $\theta$ is a homeomorphism). Suppose on the contrary that there exists $\varphi_{0} \in h(K)$ but $\varphi_{0} \notin \theta(\Delta(A))$. Let $U$ and $V$ be disjoint neighborhoods of $\varphi_{0}$ and $\theta(\Delta(A))$ respectively. By regularity of $\operatorname{reg}(M(G))$, there exist elements $\mu, \lambda \in \operatorname{reg}(M(G))$ such that $\mu^{v}\left(\varphi_{0}\right)=1, \mu^{v}\left(\Delta_{\mathrm{reg}} \backslash U\right)=0, \lambda^{v}(\theta(\Delta(A)))=1$ and $\lambda^{v}\left(\Delta_{\mathrm{reg}} \backslash V\right)=0$. It can

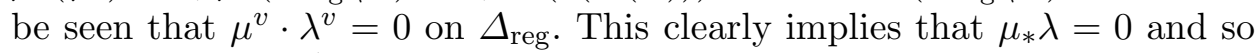
$T_{\mu} T_{\lambda}=0$. Since $\widehat{T}_{\lambda}(\Delta(A))=\lambda^{v}(\theta(\Delta(A)))=1, T_{\lambda}$ is invertible in $A$ and hence $T_{\mu}=0$. Also, since $\varphi_{0} \in h(K)$ we have $\mu^{v}\left(\varphi_{0}\right)=0$. This contradicts the fact that $\mu^{v}\left(\varphi_{0}\right)=1$. Thus $\theta(\Delta(A))=h(K)$, from which it follows that

$$
\sigma_{A}\left(T_{\mu}\right)=\mu^{v}(h(K)) \quad \text { for all } \mu \in \operatorname{reg}(M(G)) .
$$

It remains to show that $A$ is a full subalgebra of $B(X)$. Let $a \in A$ be such that $a \in B(X)^{-1}$ and let $\widetilde{A}$ be the smallest closed subalgebra of $B(X)$ that contains $a^{-1}$ and $A$. It is easily seen that $\widetilde{A}$ is commutative and $A$ is a regular subalgebra of $\widetilde{A}$. By the Shilov theorem $([7$, p. 249]) any $\varphi \in \Delta(A)$ can be extended to some $\widetilde{\varphi} \in \Delta(\widetilde{A})$. Hence since $a \in \widetilde{A}^{-1}$ we have $\varphi(a)=\widetilde{\varphi}(a) \neq 0$ for all $\varphi \in \Delta(A)$ and so $a \in A^{-1}$.

Let $\overline{\operatorname{sp}(X)}$ denote the closure of $\operatorname{sp}(X)$ in the usual topology of $\Delta_{\text {reg. }}$. Recall that $I(\overline{\operatorname{sp}(X)})$ is the largest and $J(\overline{\operatorname{sp}(X)})$ the smallest closed ideal of $\operatorname{reg}(M(G))$ whose hull is $\overline{\operatorname{sp}(X)}$.

Lemma 2.3. Under the hypotheses of Theorem 2.1,

$$
h(K)=\overline{\operatorname{sp}(X)} .
$$


Proof. It is enough to show that

$$
J(\overline{\operatorname{sp}(X)}) \subset K \subset I(\overline{\operatorname{sp}(X)}) .
$$

Let $\mu \in K$. Then $T_{\mu}=0$, which implies that $T_{\mu * f}=T_{\mu} T_{f}=0$ for all $f \in L^{1}(G)$. However since $\mu_{*} f \in L^{1}(G)$, we have $\widehat{\mu * f}=\widehat{\mu} \cdot \widehat{f}=0$ on $\operatorname{sp}(X)$ for all $f \in L^{1}(G)$, which can clearly be valid only if $\widehat{\mu}=0$ on $\operatorname{sp}(X)$. It follows that $\mu^{v}=0$ on $\overline{\operatorname{sp}(X)}$ and consequently $\mu \in I(\overline{\operatorname{sp}(X)})$. Thus we have $K \subset I(\overline{\operatorname{sp}(X)})$.

To prove $J(\overline{\operatorname{sp}(X)}) \subset K$, let $W$ be an open set in $\Delta_{\text {reg }}$ that contains $\overline{\operatorname{sp}(X)}$. Assume that $\mu^{v}$ vanishes on $W$ for some $\mu \in \operatorname{reg}(M(G))$. We have to show that $T_{\mu}=0$. First we observe that the usual topology of $\widehat{G}$ is a

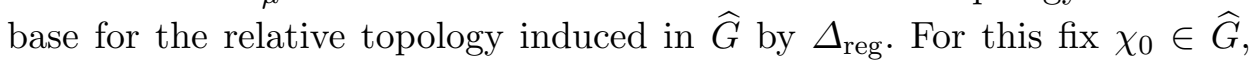
$\varepsilon>0$ and $\left\{\mu_{1}, \ldots, \mu_{n}\right\} \subset \operatorname{reg}(M(G))$. Since $\operatorname{reg}(M(G)) \supset L^{1}(G)$ it suffices to show that

$$
\begin{aligned}
U & =\left\{\chi \in \widehat{G}\left|\sup _{g \in K}\right| \chi(g)-\chi_{0}(g) \mid<\delta\right\} \\
& \subset\left\{\chi \in \widehat{G}|| \widehat{\mu}_{i}(\chi)-\widehat{\mu}_{i}\left(\chi_{0}\right) \mid<\varepsilon, i=1, \ldots, n\right\}
\end{aligned}
$$

for some compact $K \subset G$ and $\delta>0$. Choose a compact set $K$ in $G$ so that $\left|\mu_{i}\right|(G \backslash K)<\varepsilon / 4$ and $0<\delta<\varepsilon /\left(2 \max _{i}\left\|\mu_{i}\right\|\right), i=1, \ldots, n$. If $\chi \in U$, then

$$
\begin{aligned}
& \left|\widehat{\mu}_{i}(\chi)-\widehat{\mu}_{i}\left(\chi_{0}\right)\right| \leq \int_{K}\left|\chi(g)-\chi_{0}(g)\right| d\left|\mu_{i}\right|+\int_{G-K}\left|\chi(g)-\chi_{0}(g)\right| d\left|\mu_{i}\right| \\
& \quad \leq \sup _{g \in K}\left|\chi(g)-\chi_{0}(g)\right|\left(\max _{i}\left\|\mu_{i}\right\|\right)+2\left|\mu_{i}\right|(G \backslash K)<\varepsilon, \quad i=1, \ldots, n .
\end{aligned}
$$

It follows that $W \cap \widehat{G}$ is an open set in $\widehat{G}$ (in the usual topology of $\widehat{G}$ ) that contains $\operatorname{sp}(X)(=\overline{\operatorname{sp}(X)} \cap \widehat{G})$. On the other hand since $\mu^{v}=0$ on $W$, we see that $\widehat{\mu}$ vanishes on $W \cap \widehat{G}$. Now, let $\left(e_{\alpha}\right)$ be an approximate identity for $L^{1}(G)$ such that supp $\widehat{e}_{\alpha}$ is compact. Notice that $\mu_{*} e_{\alpha}$ belongs to the smallest ideal of $L^{1}(G)$ whose hull is $\operatorname{sp}(X)$. From this we deduce that $0=T_{\mu * e_{\alpha}}=T_{\mu} T_{e_{\alpha}}$. Since $T_{e_{\alpha}} x \rightarrow x$ for all $x \in X$, we conclude that $T_{\mu}=0$.

Now, we can prove the main result of this note.

Proof of Theorem 2.1. Let $\mu \in \operatorname{reg}(M(G))$. Then by Lemma 2.2, we have $\sigma\left(T_{\mu}\right)=\mu^{v}(h(K))$. On the other hand by Lemma 2.3 , since $h(K)=\overline{\operatorname{sp}(X)}$ we get $\sigma\left(T_{\mu}\right)=\mu^{v}(\overline{\operatorname{sp}(X)})$. Further, from the continuity of $\mu^{v}$ on $\Delta_{\text {reg }}$ we deduce that

$$
\mu^{v}(\overline{\operatorname{sp}(X)}) \subset \overline{\mu^{v}(\operatorname{sp}(X))}=\overline{\widehat{\mu}(\operatorname{sp}(X))} .
$$

Also since $\overline{\operatorname{sp}(X)}$ is a compact subset of $\Delta_{\text {reg }}$, it follows that $\mu^{v}(\overline{\operatorname{sp}(X)})$ is closed and consequently

$$
\mu^{v}(\overline{\operatorname{sp}(X)}) \supset \overline{\mu^{v}(\operatorname{sp}(X))}=\overline{\widehat{\mu}(\operatorname{sp}(X))} .
$$


Thus, we obtain

$$
\sigma\left(T_{\mu}\right)=\overline{\widehat{\mu}(\operatorname{sp}(X))}
$$

The proof is complete.

Let $Y$ be a Banach $M(G)$-submodule of $X$. Define $\operatorname{sp}(Y)$ as the hull in $L^{1}(G)$ of the ideal $I_{Y}=\left\{f \in L^{1}(G) \mid T_{f} y=0, y \in Y\right\}$.

COROLlary 2.4. Assume the hypotheses of Theorem 2.1 are satisfied. If $Y$ is a Banach $M(G)$-submodule of $X$, then

$$
\sigma\left(T_{\mu} \mid Y\right)=\overline{\widehat{\mu}(\operatorname{sp}(Y))} \quad \text { for all } \mu \in \operatorname{reg}(M(G)) \text {. }
$$

\section{References}

[1] E. Albrecht, Decomposable Systems of Operators in Harmonic Analysis, Birkhäuser, Basel, 1982, 19-35.

[2] W. Arveson, On groups of automorphisms of operator algebras, J. Funct. Anal. 15 (1974), 217-243.

[3] N. Bourbaki, Théories spectrales, Hermann, 1967.

[4] A. Connes, Une classification des facteurs de type III, Ann. Sci. École Norm. Sup. 6 (1973), 133-152.

[5] C. D'Antoni, R. Longo and L. Zsidó, A spectral mapping theorem for locally compact groups of operators, Pacific J. Math. 103 (1982), 17-24.

[6] J. Eschmeier, Operator decomposability and weakly continuous representations of locally compact abelian groups, J. Operator Theory 7 (1982), 201-208.

[7] R. Larsen, Banach Algebras, Dekker, New York, 1973.

[8] M. A. Rieffel, Induced Banach representations of Banach algebras and locally compact groups, J. Funct. Anal. 1 (1967), 443-491.

[9] W. Rudin, Fourier Analysis on Groups, Interscience, New York, 1962.

[10] H. Seferoğlu, Spectral mapping theorem for representations of measure algebras, Proc. Edinburgh Math. Soc. 40 (1997), 261-266.

[11] S.-E. Takahasi and J. Inoue, A spectral mapping theorem for some representations of compact abelian groups, ibid. 35 (1992), 47-52.

[12] W. Żelazko, Banach Algebras, PWN and Elsevier, Warszawa, 1973.

Department of Mathematics

Faculty of Art and Science

Yüzüncü Yil University

65080 Van, Turkey

E-mail: seferoglu2003@yahoo.com

Received June 29, 1998

Revised version December 5, 2002 